\title{
A Finite Volume Procedure for Fluid Flow, Heat Transfer and Solid-Body Stress
}

\author{
P. I. Jagad, B. P. Puranik \& A. W. Date
}

\begin{abstract}
A unified cell-centered unstructured mesh finite volume procedure is presented for fluid flow, heat transfer and solid-body stress analysis. An in-house procedure (A. W. Date, Solution of Transport Equations on Unstructured Meshes with Cell-Centered Colocated Variables. Part I: Discretization, International Journal of Heat and Mass Transfer, vol. 48 (6), 1117-1127, 2005) is extended to include the solid-body stress analysis. The transport terms for a cell-face are evaluated in a structured grid-like manner. The Cartesian gradients at the center of each cell-face are evaluated using the coordinate transformation relations. The accuracy of the procedure is demonstrated by solving several benchmark problems involving different boundary conditions, source terms, and types of loading.

Keywords: cell-centered, finite volume procedure, fluid flow, heat transfer, solid-body stress, unstructured mesh.
\end{abstract}

\section{Introduction}

A finite volume (FV) discretization procedure consists of using the integral form of the governing equations for each arbitrary, contiguous, finite volume or cell comprising the mesh used to map the computational domain. This procedure is of interest for solving continuum mechanics problems because of its ability to satisfy the conservation principles of a continuum field locally, within each of the cells used to discretize the computational domain, as well as on the global scale across the whole domain [1]. Furthermore, it ensures continuity of the field 
quantities and their derivatives across the cell boundaries [2]. The FV procedure has been predominantly used for solving non-linear fluid flow problems over the last four decades. When dealing with fluid-structure interaction (FSI) problems [3, 4], the FV discretization procedure is convenient to use for solving governing equations in the solid as well. The advancement in unstructured mesh FV procedures during last two decades has generated sufficient interest in using it for solving solid mechanics problems as well $[5,6,7]$, as complex geometries can be mapped conveniently using the unstructured mesh.

In most previous cell-centered unstructured mesh FV procedures $([6,8,9])$, the diffusive transport for a face is evaluated as

$$
D_{k} \approx \mu_{\phi, c k}\left(\frac{\partial \phi}{\partial x_{j}}\right)_{c k} n_{j, c k} A_{f k}
$$

where $\phi$ stands for a displacement component $\delta u_{i}, \mu$ is the diffusion coefficient, $n_{j}$ is the outward facing unit normal to the face, $A_{f k}$ is the face area and the subscript ck stands for the center of the face - $k$. The Cartesian gradients $\left(\partial \phi / \partial x_{j}\right)_{c k}$ in Eq. (1) are approximated as

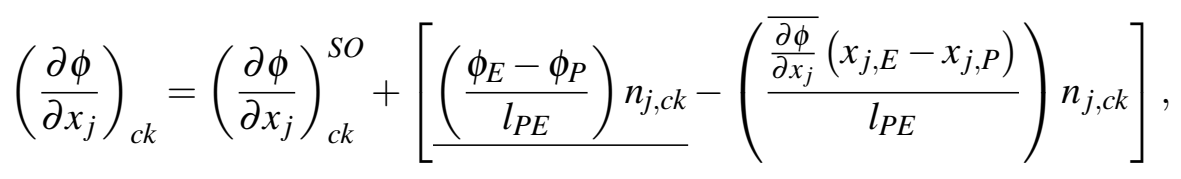

where

$$
\left(\frac{\partial \phi}{\partial x_{j}}\right)_{c k}^{S O}=\frac{1}{2}\left[\left(\frac{\partial \phi}{\partial x_{j}}\right)_{P}+\left(\frac{\partial \phi}{\partial x_{j}}\right)_{E}+\left(\frac{\partial}{\partial x_{l}}\left(\frac{\partial \phi}{\partial x_{j}}\right)\right)_{P}\left(x_{l, c k}-x_{l, P}\right)+\left(\frac{\partial}{\partial x_{l}}\left(\frac{\partial \phi}{\partial x_{j}}\right)\right)_{E}\left(x_{l, c k}-x_{l, E}\right)\right], l=1,2,3
$$

and

$$
\overline{\frac{\partial \phi}{\partial x_{j}}}=\frac{1}{2}\left[\left(\frac{\partial \phi}{\partial x_{j}}\right)_{P}+\left(\frac{\partial \phi}{\partial x_{j}}\right)_{E}\right]
$$

where $l_{P E}$ is the distance between the cell centers $P$ and $E$ straddling the face, and $x_{j}$ stands for the Cartesian coordinate vector. The Cartesian gradients $\partial \phi / \partial x_{j}$ at the cell centers $P$ and $E$ are evaluated using a least squares based procedure [10]. The additional terms in [ ] brackets in Eq. (2) are employed for stability. The terms in Eq. (2) other than the one which 
is underlined are added to the source term and are evaluated explicitly. The underlined term is evaluated implicitly and hence it is used for the discrete representation of the diffusive transport for the face.

One of the procedures $([11,12])$ evaluates the diffusive transport through a face - $k$ as

$$
D_{k}=\mu_{\phi, c k}\left(\frac{\partial \phi}{\partial x_{j}}\right)_{c k} A_{j, f k}
$$

The scalar product $\left(\partial \phi / \partial x_{j}\right)_{c k} A_{j, f k}$ in Eq. (5) is evaluated using transformation from physical coordinates $(x, y)$ to computational coordinates $(\xi, \eta)$. The expression is

$$
\left(\frac{\partial \phi}{\partial x_{j}}\right)_{c k} A_{j, f k}=\left[\left(\frac{\phi_{E}-\phi_{P}}{l_{P E}}\right) \frac{A_{j, f k} A_{j, f k}}{A_{j, f k} e_{j, s}}\right]_{k}+\left[\left(\frac{\phi_{b}-\phi_{a}}{\left|A_{f k}\right|}\right) \frac{A_{j, f k} A_{j, f k}}{A_{j, f k} e_{j, s}} e_{j, t} e_{j, s}\right]_{k},
$$

where $a$ and $b$ stand for the vertices of the face. The unit vectors $e_{j, s}$ (along the line joining the cell centers $P$ and $E$ ) and $e_{j, t}$ (tangent vector along the face) are expressed as

$$
\begin{gathered}
e_{j, s}=\frac{x_{j, E}-x_{j, P}}{d S}, \\
e_{j, t}=\frac{x_{j, b}-x_{j, a}}{\left|A_{f k}\right|} .
\end{gathered}
$$

The first term on the right hand side of Eq. (6) represents the primary gradient and is treated implicitly. The second term on the right hand side of Eq. (6) is secondary or cross diffusion term, which is equal to zero on orthogonal grids. This term is evaluated by expressing it as the difference between the total diffusion and the primary component. Two different formulations are considered for the evaluation of the transpose and the divergence parts of the stress tensor. In the first case, these terms are evaluated explicitly using a procedure identical to the one employed in $[6,8,9]$. In the second case, the stress tensor is linearized completely for implicit 
evaluation of the transpose and divergence terms, and enhance the convergence rate.

In Davidson [13], the Cartesian gradients $\left(\partial \phi / \partial x_{j}\right)_{c k}$ in Eq. (1) are evaluated by assuming a control volume surrounding the face center and using the divergence theorem. For example

$$
\left(\frac{\partial \phi}{\partial x_{i}}\right)_{c k}=\frac{1}{\triangle V_{c k}} \int_{A} \phi n_{i} d A,
$$

where $\triangle V_{c k}$ stands for the volume surrounding the face center and $A$ stands for the total surface area enclosing this volume. Dalal et al. [14] evaluates the scalar product $\left(\partial \phi / \partial x_{j}\right)_{c k} n_{j, c k}$ as follows.

$$
\left(\frac{\partial \phi}{\partial x_{j}}\right)_{c k} n_{j, c k}=\left(\frac{\partial \phi}{\partial x_{j}}\right)_{c k}\left(n_{j, 1}+n_{j, 2}\right)
$$

where $n_{j, 1}$ is a unit vector joining the cell center $P$ with the neighbouring cell center $E$, and $n_{j, 2}=n_{j, c k}-n_{j, 1}$. Equation (10) is rewritten as

$$
\left(\frac{\partial \phi}{\partial x_{j}}\right)_{c k} n_{j, c k}=\left(\frac{\partial \phi}{\partial x_{j}}\right)_{c k} n_{j, 1}+\left(\frac{\partial \phi}{\partial x_{j}}\right)_{c k} n_{j, 2}
$$

or

$$
\left(\frac{\partial \phi}{\partial x_{j}}\right)_{c k} n_{j, c k}=\frac{\phi_{E}-\phi_{P}}{d S}+\left[\frac{\triangle V_{E}\left(\frac{\partial \phi}{\partial x_{j}}\right)_{P}+\triangle V_{P}\left(\frac{\partial \phi}{\partial x_{j}}\right)_{E}}{\triangle V_{E}+\triangle V_{P}}\right] n_{j, 2}
$$

where $\triangle V$ stands for the cell volume. The second term (the term in [ ] brackets) on the right hand side of Eq. (12) represents the cross diffusion.

Traore et al. [15] proposed an iterative method for the evaluation of the diffusion transport for a cell-face, which was called the improved deferred correction. In this method, the unit normal to a cell face is resolved into the following two components.

$$
\vec{n}_{k}=\lambda_{k} \vec{\xi}_{k}+\beta_{k} \vec{t}_{k}
$$

where $\lambda_{k}=1 / \cos \theta_{k}$ and $\beta_{k}=\tan \theta_{k}$. Here $\cos \theta_{k}$ is the projection of the cell face normal $\vec{n}_{k}$ onto $\vec{\xi}_{k}$ and the projection of $\vec{t}_{k}$ onto $\vec{\xi}_{k}$ is $-\sin \theta_{k}$. The transport for the cell is now expressed as 


$$
\sum_{k=e, w, n, s} \lambda_{k} A_{f k}(\nabla \phi)_{k}^{m} \cdot \vec{\xi}_{k}=(g)_{P} \cdot V_{P}-\sum_{k=e, w, n, s} \beta_{k} A_{f k}(\nabla \phi)_{k}^{m-1} \cdot \vec{t}_{k}
$$

The left hand side of (14) is approximated by a centered formula of second order. The second term of the right hand side is explicitly determined from the gradient at nodal values of the previous iteration interpolated at the $k$ location. The gradient at nodal values is computed with the Gauss formula.

To discretize the diffusive transport for a cell face, Xue and Barton [16] performed a vector decomposition along a virtual line that passes through the midpoint of the face and is parallel to the line connecting the centroid of the cells sharing the face. The unit normal to the cell face can be decomposed as

$$
\vec{n}_{k}=\alpha_{k} \vec{d}_{k}+\gamma_{k} \vec{t}_{k}
$$

where $\alpha_{k} \vec{d}_{k}$ and $\gamma_{k} \vec{t}_{k}$ are vectors along the virtual line and along the tangent to the face respectively. The gradient along the normal direction at the midpoint of the face can be expressed as

$$
\left(\nabla \phi \cdot \vec{n}_{k}\right)=\frac{\alpha_{k}}{d s_{k}}\left(\phi_{N B}-\phi_{P}\right)+\frac{\alpha_{k}}{d s_{k}}\left(\nabla \phi_{N B}-\nabla \phi_{P}\right) \cdot r_{f-f}+\gamma_{k} \nabla \phi_{k} \cdot \vec{t}_{k}
$$

for an interior face. Here $d s_{k}$ is the distance from cell centroid $P$ to its neighbouring cell centroid NB and $r_{f-f}$, is the distance between the virtual line and the line connecting the cell centroids $P$ and $N B$.

In the present work, a cell-centered unstructured mesh FV procedure is presented for fluidflow, heat transfer and solid-body stress analysis. The procedure of Date [17] is extended to include the solid-body stress analysis with an objective of making the procedure suitable to be adapted in a monolithic approach [18] for the solution of FSI problems. The evaluation of the transport terms (convection and diffusion) for a cell-face is performed along an assumed normal line passing through the face-center (in a structured grid-like manner). Thus, discretization of the face transport is always ensured along an orthogonal line passing through the center / 
mid-point of the face which is consistent with the use of mid-point rule for the evaluation of the face integral. This ensures that the solution accuracy is not affected by the topology (skewness) of the mesh [16]. The notion of smoothing pressure correction [19] is employed for eliminating the prediction of zig-zag or checker-board type of pressure filed on the meshes with co-located variables. To compute the solid body stress, the terms in the stress tensor that can not be accommodated in the diffusion term, i.e., the transpose and the divergence terms are required to be evaluated. For the evaluation of these terms, conventionally [5, 6, 7, 8, 9], the face center Cartesian gradients $\left(\partial \phi / \partial x_{j}\right)_{c k}$ are approximated through the Taylor series expansion at the cell-centers straddling the face and then using the arithmetic mean of the two expanded values. This requires an additional computation of the second order gradients $\left(\partial\left(\partial() / \partial x_{i}\right) / \partial x_{j}\right)$ at the cell centers, i.e., at $P$ and E. (see Eq. 22) In the present work, the transformation relations between the global Cartesian and local curvilinear coordinates are employed for the said purpose, which eliminates such a requirement of computation of second order gradients at the cell centers. The accuracy of the present procedure is demonstrated by solving a few benchmark problems comprising of fluid flow, heat transfer and solid-body stress analysis.

\section{The Discretization Procedure}

The velocity field is determined using the principle of conservation of linear momentum of the fluid flow. The SIMPLE [20] algorithm is used for evaluation of the pressure field via the continuity equation. Thus, the pressure correction equation is derived from the continuity equation. A displacement formulation of the equilibrium equations constitutes the governing differential equations for the solid for the determination of the displacement field. The stress field is then derived from the displacement field using the constitutive relations. Energy equation is used as the governing differential equation for the determination of the temperature field. The differential equation governing the conservation laws, under steady state, for the fluid as well as the solid can be written as 


$$
a_{\phi}\left[\frac{\partial\left(\rho_{f} u_{j} \phi\right)}{\partial x_{j}}\right]=\frac{\partial}{\partial x_{j}}\left[\Gamma_{\phi}\left(\frac{\partial \phi}{\partial x_{j}}\right)\right]+S_{\phi},
$$

where $\phi$ stands for the velocity component $\left(u_{i}\right)$ or the temperature of the fluid $\left(T_{f}\right)$ or the temperature of the solid $\left(T_{s}\right)$ or displacement component $\left(\delta u_{i}\right), \rho_{f}$ stands for the density of the fluid, $u_{j}$ stands for the velocity vector, $\Gamma_{\phi}$ stands for the diffusion coefficient corresponding to the variable $\phi, S_{\phi}$ stands for the source term corresponding to the variable $\phi$. Expressions of $a_{\phi}, \Gamma_{\phi}$ and $S_{\phi}$ are given in Table 1 for different interpretations of $\phi$, where incompressible fluid and linear thermo-elastic solid are assumed. For $\phi=1$, Eq. (17) represents continuity equation. In Table $1, \mu_{f}$ stands for the dynamic viscosity of the fluid, $c_{p, f}$ stands for the specific heat of the fluid at constant pressure, $k_{f}$ and $k_{s}$ stand for the thermal conductivity of the fluid and that of solid respectively, $p$ stands for the fluid pressure, $B_{i, f}$ and $B_{i, s}$ stand for the body force per unit volume in direction - $i$ for the fluid and the solid respectively, $Q^{\prime \prime \prime}$ stands for the source of heat per unit volume, $\alpha_{s}$ stands for the coefficient of linear thermal expansion of the solid, $T_{r}$ stands for the reference temperature corresponding to zero thermal stress condition, and $\delta_{i j}$ stands for the Kronecker delta. For a homogeneous and isotropic material, the shear modulus $\mu_{s}=E /[2(1+v)]$ and the Lame's constant $\lambda_{s}=E v /[(1+v)(1-2 v)]$. Here, $E$ is the Young's modulus and $v$ is the Poisson's ratio of the material.

Integrating Eq. (17) over an arbitrary control volume (CV) or cell comprising the computational mesh we get

$$
a_{\phi} \int_{\triangle V}\left[\frac{\partial\left(\rho_{f} u_{j} \phi\right)}{\partial x_{j}}\right] d V=\int_{\triangle V}\left[\frac{\partial}{\partial x_{j}}\left[\Gamma_{\phi}\left(\frac{\partial \phi}{\partial x_{j}}\right)\right]\right] d V+\int_{\triangle V}\left[S_{\phi}\right] d V
$$

where $\triangle V$ is the volume of the cell. The term on the left hand side in Eq. (18) represents convective transport and the first and second terms on the right hand side represent diffusive transport and source respectively. The discretization of each of these terms is carried out according to the procedure of Date et al. [17] and the additions only are presented here. 


\subsection{Equilibrium Equation}

The terms in the stress tensor that can not be accommodated in the diffusion term, i.e., the transpose, divergence, and thermal stress terms [21] are added to the source term (along with the body force term if any) and are evaluated explicitly. The expanded form of the source term is

$$
\begin{gathered}
\int_{\triangle V}\left(S_{\phi}\right) d V=\int_{\triangle V} B_{i, s} d V+ \\
\int_{\triangle V}\left[\frac{\partial}{\partial x_{j}}\left[\mu_{s}\left(\frac{\partial\left(\delta u_{j}\right)}{\partial x_{i}}\right)+\lambda_{s} \delta_{i j} \frac{\partial\left(\delta u_{l}\right)}{\partial x_{l}}-\left(3 \lambda_{s}+2 \mu_{s}\right) \alpha_{s} \delta_{i j}\left(T_{s}-T_{r}\right)\right]\right] d V .
\end{gathered}
$$

The volume integral of the body force term in Eq. (19) is approximated using the mid-point rule. The remaining terms are evaluated using the divergence theorem and the mid-point rule. Therefore

$$
\begin{gathered}
\int_{\triangle V}\left(S_{\phi}\right) d V=B_{i, s} \Delta V+ \\
\sum_{k=1}^{N k}\left[\mu_{s}\left(\frac{\partial\left(\delta u_{j}\right)}{\partial x_{i}}\right)+\lambda_{s} \delta_{i j} \frac{\partial\left(\delta u_{l}\right)}{\partial x_{l}}-\left(3 \lambda_{s}+2 \mu_{s}\right) \alpha_{s} \delta_{i j}\left(T_{s}-T_{r}\right)\right]_{c k} n_{j, c k} A_{f k} .
\end{gathered}
$$

Equation (20) can be rewritten as

$$
\begin{gathered}
\int_{\triangle V}\left(S_{\phi}\right) d V=B_{i, s} \Delta V+\sum_{k=1}^{N k}\left[\mu_{s}\left(\frac{\partial\left(\delta u_{j}\right)}{\partial x_{i}}\right) n_{j}\right]_{c k} A_{f k}+ \\
\sum_{k=1}^{N k}\left[\lambda_{s} \frac{\partial\left(\delta u_{l}\right)}{\partial x_{l}} n_{i}\right]_{c k} A_{f k}-\sum_{k=1}^{N k}\left[\left(3 \lambda_{s}+2 \mu_{s}\right) \alpha_{s}\left(T_{s}-T_{r}\right) n_{i}\right]_{c k} A_{f k} .
\end{gathered}
$$


Conventionally [6, 8, 9], the Cartesian gradients at the face-centers in Eq. (21) are evaluated through the Taylor series expansion at the cell-centers straddling the face and then using the arithmetic mean of the two expanded values. For example, the Cartesian gradient of the displacement component $\delta u_{i}$ in coordinate direction - $k$ is evaluated as

$$
\frac{\partial\left(\delta u_{i}\right)}{\partial x_{k}}=\left[\left(\frac{\partial\left(\delta u_{i}\right)}{\partial x_{k}}\right)_{P}+\left(\frac{\partial}{\partial x_{j}}\left(\frac{\partial\left(\delta u_{i}\right)}{\partial x_{k}}\right)\right)_{P}\left(x_{j, c k}-x_{j, P}\right)+\left(\frac{\partial\left(\delta u_{i}\right)}{\partial x_{k}}\right)_{E}+\left(\frac{\partial}{\partial x_{j}}\left(\frac{\partial\left(\delta u_{i}\right)}{\partial x_{k}}\right)\right)_{E}\left(x_{j, c k}-x_{j, E}\right)\right] .
$$

In the present method a direct evaluation of the Cartesian gradients at the face-centers is proposed by using the local curvilinear coordinate system (Fig. 1) for each cell-face. Using the formula for the coordinate transformation between the local curvilinear coordinates $\xi_{j}$ and the global Cartesian coordinates $x_{j}$ we have

$$
\frac{\partial\left(\delta u_{i}\right)}{\partial x_{k}}=\frac{1}{J}\left[\beta_{j}^{k} \frac{\partial\left(\delta u_{i}\right)}{\partial \xi_{j}}\right]
$$

where $J$ is the Jacobin of the coordinate transformation matrix which is expressed as

$$
J=\frac{\partial x_{1}}{\partial \xi_{j}} \beta_{j}^{1}
$$

where $\beta_{j}^{k}$ are the geometric coefficients expressed as

$$
\beta_{j}^{k}=\frac{\partial x_{p}}{\partial \xi_{r}} \frac{\partial x_{q}}{\partial \xi_{s}}-\frac{\partial x_{q}}{\partial \xi_{r}} \frac{\partial x_{p}}{\partial \xi_{s}}
$$

Here $k, p, q$ are cyclic and $j, r, s$ are cyclic. Consider the cell-face ABC common between the two tetrahedral cells $A B C D$ and $A B C F$ as shown in Fig. 1. Using the second order accurate Taylor series expansion, the gradients with respect to the local coordinates $\xi_{j}$ in E. (23) are expressed as 


$$
\begin{aligned}
& \frac{\partial\left(\delta u_{i}\right)}{\partial \xi_{1}}=\frac{\delta u_{i, E}-\delta u_{i, P}}{l_{P E}}, \\
& \frac{\partial\left(\delta u_{i}\right)}{\partial \xi_{2}}=\frac{\delta u_{i, A}-\delta u_{i, C}}{l_{A C}}, \\
& \frac{\partial\left(\delta u_{i}\right)}{\partial \xi_{3}}=\frac{\delta u_{i, B}-\delta u_{i, C}}{l_{B C}} .
\end{aligned}
$$

The value of $\delta u_{i}$ at the face vertices $A, B$ and $C$ in Eqs. (27) and (28) can be expressed as

$$
\phi_{n}=\frac{1}{2}\left[\phi_{P}+\left(x_{j, n}-x_{j, P}\right)\left(\frac{\partial \phi}{\partial x_{j}}\right)_{P}+\phi_{E}+\left(x_{j, n}-x_{j, E}\right)\left(\frac{\partial \phi}{\partial x_{j}}\right)_{E}\right] .
$$

where $\phi$ stands for the displacement component $\delta u_{i}$ and the suffix $n$ stands for the vertex $A$ or B or $C$.

\subsection{Boundary Conditions}

If the displacement $\delta u_{i, B}$ is known at the boundary node $B$, it is directly substituted into the discretized governing equation for the near boundary cell.

If the forces per unit area or traction $t_{i, B}$ is known at the boundary node $B$, the force acting on the boundary is computed from the known traction. This force is then used in the implementation of the force balance for the adjacent cell to the boundary.

Implementation of symmetry boundary condition is identical to that for the velocity [17].

\section{Test Problems}

In order to demonstrate the accuracy of the present procedure, a few fluid flow and heat transfer as well as solid-body stress analysis benchmark problems are solved. The accuracy is verified 
for different boundary conditions as well as for the presence of source term by comparing the results with the available analytical, numerical and experimental results in the literature. For the solution of solid-body stress analysis test problems, meshes consisting partly of skewed cells are employed in order to demonstrate the accuracy of the procedure on such meshes. The solution procedure of Date [17] is extended to include the additions mentioned in Section 2.1.

\subsection{Fluid Flow and Heat Transfer Problems}

Steady, laminar flow is considered. The fluid properties are assumed to be constant. Solution of these problems consists of solving one or more of the following dimensionless equations. They represent specific dimensionless forms of the general governing equation (Eq. (17)), written here for convenience.

Continuity equation:

$$
\frac{\partial u_{j}^{*}}{\partial x_{j}^{*}}=0
$$

Momentum equations:

$$
\frac{\partial\left(u_{i}^{*} u_{j}^{*}\right)}{\partial x_{j}^{*}}=-\frac{\partial p^{*}}{\partial x_{i}^{*}}+\Gamma_{u_{i}}^{*}\left(\frac{\partial^{2} u_{i}^{*}}{\partial x_{j}^{* 2}}\right)+B_{i}^{*}
$$

Energy equation:

$$
\frac{\partial\left(T^{*} u_{j}^{*}\right)}{\partial x_{j}^{*}}=\Gamma_{T}^{*}\left(\frac{\partial^{2} T^{*}}{\partial x_{j}^{* 2}}\right)
$$

The scales used for dimension-less representation of the variables in Eqs. (30), (31) and (32) are specified in the formulation of each problem. The expression of dimensionless diffusion coefficients $\Gamma_{u_{i}}^{*}$ and $\Gamma_{T}^{*}$ in Eqs. (31) and (32) respectively and that of the dimension-less body force in direction - $i\left(B_{i}^{*}\right)$ in Eq. (31) are also specified in the formulation of the problems. 


\section{Natural Convection in a Horizontal Concentric Annulus}

The problem of natural convection in an annular cavity between horizontal concentric cylinders is considered as shown in Fig. 2. The vertical plane through the center of the system divides the flow into two symmetric halves, so that only one side (left) is employed for the simulations. The ratio of the annulus gap width to the outside diameter of the inner cylinder, i.e., $\left[\left(D_{o}-D_{i}\right) / 2\right] / D_{i}$ is assumed to be 0.8 . Here $D_{o}$ stands for the inner diameter of the outer cylinder and $D_{i}$ stands for the outer diameter of the inner cylinder. The annulus gap width $\left(D_{o}-D_{i}\right) / 2$ is used as the characteristic length scale $\left(L_{r e f}\right)$ and $\alpha_{f} / L_{r e f}$ is used as the characteristic velocity scale, where $\alpha_{f}$ stands for the thermal diffusivity. The continuity equation, the $x$ and $y$ - momentum equations and the energy equation are solved. The diffusion coefficients $\Gamma_{u_{i}}^{*}$ and $\Gamma_{T}^{*}$ are assumed to be equal to $\operatorname{Pr}=v_{f} / \alpha_{f}$ (Prandtl number) and 1 respectively, where $v_{f}=\rho_{f} / \mu_{f}$ stands for the kinematic viscosity. The expression of $B_{1}^{*}$ in the $x$ - momentum equation is assumed to be zero and that of $B_{2}^{*}$ in $y$ - momentum equation is assumed to be equal to $\operatorname{RaPr} T^{*}$. Here $\operatorname{Ra}=g \beta_{f}\left(T_{h}-T_{c}\right) L_{r e f}^{3} / \nu_{f} \alpha_{f}$ stands for the Rayleigh number, where $g$ stands for the acceleration due to gravity and $\beta_{f}$ stands for the coefficient of volumetric thermal expansion. No-slip boundary condition is assumed on the inner and outer walls. The inner and outer walls are assumed to be isothermal at temperatures $T_{h}$ and $T_{c}$ respectively. Hence, the dimensionless temperatures $T^{*}=\left(T-T_{c}\right) /\left(T_{h}-T_{c}\right)$ at the inner and outer walls are 1.0 and 0 respectively. The normal gradients of temperature and that of the tangential component of the velocity $(v)$ are assumed to be zero at the symmetry boundaries. The normal component of the velocity $(u)$ at the symmetry boundaries is assumed to be zero. The flow within the annulus is induced by the buoyancy force which results due to the differential heating of the cylinders. A fluid with Prandtl number 0.7 is considered. The problem is solved at three different values of Rayleigh number: 100,1000 and 10,000. A representative mesh is shown in Fig. 3. The UDS advection scheme is used. The results of these problems are usually reported in terms of an equivalent conductivity $\left(k_{e q}\right)$, which is defined as the actual heat flux (transferred to the fluid from the wall or from the fluid to the wall at a point on the wall) divided by the heat flux that would occur by pure conduction in the absence of fluid motion. Thus, for inner cylinder $k_{e q}=q_{w, i} D_{i} \ln \left(D_{o} / D_{i}\right) /\left[2 k_{f}\left(T_{h}-T_{c}\right)\right]$ and for outer cylinder 
$k_{e q}=q_{w, o} D_{o} \ln \left(D_{o} / D_{i}\right) /\left[2 k\left(T_{h}-T_{c}\right)\right]$. The distribution of $k_{e q}$ along the inner and outer wall surfaces (assuming $\theta=0^{\circ}$ at the top) is shown in Fig. 4 and the mean values are reported in Table 2. A comparison of the results with that of [22] shows a good agreement.

\section{Flow Over a Backward Facing Step}

As shown in Fig. 5, the computational domain consists of a rectangular region of height $H$ and length $30 H$. The height $H$ is used as the characteristic length scale. At the beginning of this region a step of height $H / 2$ is provided. A fully developed flow enters the region through the inlet of height $H / 2$ above the step. The continuity and the $x$ and $y$-momentum equations are solved. The expressions of $\Gamma_{u_{i}}^{*}$ and $B_{i}^{*}$ in the momentum equations are set equal to $1 / R e$ and zero respectively. Both velocity components are assumed zero at all solid walls including the step. It is assumed that the flow becomes fully developed at the exit as sufficient length of the channel is considered. Normal gradient of all dependent variables is assumed equal to zero at the exit. A standard test case of $R e$ equal to 800 is considered. Here $R e=\rho_{f} \bar{u}_{\text {in }} H / \mu_{f}$, where $\bar{u}_{\text {in }}$ stands for the average velocity at the inlet which is used as the characteristic velocity scale. A triangular mesh (5760 elements) as shown in Fig. 6 is used for computations. An extract from the complete mesh (along the length) is shown for clarity. A CDS dominating blend (0.9 CDS ) of UDS and CDS is used as the advection scheme. The streamlines are shown in Fig. 7. The flow entering above the step separates from the lower wall downstream of the step and then reattaches to the lower wall, forming a recirculation zone in the step corner. The length of this zone is calculated to be $6.03 H$ against $5.94 H$ reported in [23]. Due to sudden expansion at the inlet, the pressure of the fluid decreases. Recovery of the pressure takes place along the downstream up to a certain length. In this region, the flow separates from the upper wall due to adverse pressure gradient. Further downstream, the flow reattaches. This forms another recirculation zone. The length of this zone is calculated to be $5.53 \mathrm{H}$ against $5.51 \mathrm{H}$ reported in [23]. Profiles of $x$ - velocity component at different axial locations are shown in Fig. 8. These are found to be in good agreement with that reported in [23]. 


\section{Lid Driven Cubic Cavity}

As shown in Fig. 9, a 3-dimensional problem of lid driven flow in a cubic cavity of side $L$ is considered. The length $L$ is used as the characteristic length scale for this problem. The continuity and $x, y$ and $z$ - momentum equations are solved. The expressions of the $\Gamma_{u_{i}}^{*}$ and $B_{i}^{*}$ in the momentum equations are set equal to $1 / R e$ and zero respectively. At the four vertical walls and the bottom wall, no slip boundary condition is assumed. The top wall is assumed to be moving with a constant velocity $V_{\text {lid }}$ in the positive $x$ - direction. This circulates the fluid within the cavity due to shear. The velocity of the lid $V_{l i d}$ is used as the characteristic velocity scale. A tetrahedral mesh with finer mesh near the walls is employed. The profile of $x$-velocity at $x^{*}=z^{*}=0.5$ is shown in Fig. 10 for $R e=\rho_{f} V_{l i d} L / \mu_{f}=400$. The results are compared with that of Jiang et al. [24] and good agreement is found.

\subsection{Solid-body Stress Analysis Problems}

\section{Flat Plate with a Circular Hole Subjected to Uniform Tension}

A case of an infinite flat plate with a small circular hole in its center and subjected to a uniform tension in one direction is considered. The schematic of the problem is shown in Fig. 11. For this problem an analytical solution is available [25] and is given by

$$
\begin{gathered}
\sigma_{x x}=t_{x}\left[1-\frac{a^{2}}{r^{2}}\left(\frac{3}{2} \cos 2 \theta+\cos 4 \theta\right)+\frac{3}{2} \frac{a^{4}}{r^{4}} \cos 4 \theta\right], \\
\sigma_{y y}=t_{x}\left[-\frac{a^{2}}{r^{2}}\left(\frac{1}{2} \cos 2 \theta-\cos 4 \theta\right)-\frac{3}{2} \frac{a^{4}}{r^{4}} \cos 4 \theta\right], \\
\sigma_{x y}=t_{x}\left[-\frac{a^{2}}{r^{2}}\left(\frac{1}{2} \sin 2 \theta+\sin 4 \theta\right)+\frac{3}{2} \frac{a^{4}}{r^{4}} \sin 4 \theta\right],
\end{gathered}
$$

where $a$ is radius of the hole and $r=\left(x^{2}+y^{2}\right)^{1 / 2}$ and $\theta=\tan ^{-1}(y / x)$ are the usual polar coordinates.

To solve the problem numerically, finite dimensions of the plate are considered such that the hole is still considered to be small. One quarter of the domain, i.e., $\mathrm{ABCDE}$ is used due 
to symmetry. At the top and right edges, $\mathrm{CD}$ and $\mathrm{BC}$ respectively, of the domain the stresses obtained using the analytical solution are used as the boundary condition. Symmetry boundary condition is used at the left and bottom edges, i.e., at $\mathrm{DE}$ and $\mathrm{AB}$ respectively. The edge $\mathrm{AE}$ is assumed to be traction free. The value of the uniform tension $t_{x}$ in the $\mathrm{x}$-direction is assumed to be $10^{4} \mathrm{~Pa}$. The material properties assumed are $\mathrm{E}=10^{7} \mathrm{~Pa}$ and $v=0.3$. An initial mesh consisting of 1224 skewed (in some part of the mesh) triangular cells (see Fig. 12) is used. Average error in computation of $\sigma_{x x}, \sigma_{y y}$ and $\sigma_{x y}$ is found to be 1.3, 4.7 and 3.3 percent respectively. Definition of the average error as given in [6] is used, i.e., the average error is found by summing the weighted absolute values of differences between the predicted stresses and that given by Eqs. (33), (34), and (35). The weighting factors are calculated as the ratio of the cell volume and the total volume of the computational domain. The error is normalized with the average value of the corresponding stress in the computational domain. Without changing the topology of the cells, the mesh was refined and the average errors for a mesh consisting of 8704 triangular cells is found to be $0.6,2.0$ and 1.4 percent in computation of $\sigma_{x x}, \sigma_{y y}$ and $\sigma_{x y}$ respectively. The overlapping plots of the contours of stress components in the plate calculated using the present procedure and those obtained using the analytical solutions are shown in Fig. 13.

\section{A Cube Subjected to a Uniform Temperature Rise}

If a body is subjected to a uniform temperature rise $\Delta T=T-T_{r}$, with $T_{r}$ as the reference temperature corresponding zero thermal stress condition, then in the absence of body forces the analytical solutions are available for the following two types of boundary conditions[26].

1. The body is free to expand. In this case the displacements are linear functions of the space vector $\left(\delta u_{i}=\alpha_{s} \triangle T x_{i}\right)$ and all stress components are zero.

2. The surfaces are fixed such that all displacement components are zero on the boundaries. This results into zero displacement field in the body and a uniform stress field $\sigma_{i j}=$ $-\left(3 \lambda_{s}+2 \mu_{s}\right) \alpha_{s} \triangle T \delta_{i j}$

The schematic of the problem for the first case, when the cube is assumed to be free to expand, is shown in Fig. 14. Only one eighth of the cube is used as a solution domain considering the 
symmetry. It is assumed that the cube is subjected to a uniform temperature rise of $\triangle T=100 \mathrm{~K}$ and $\alpha_{s}=1 \times 10^{-5} K^{-1}$. A skewed prismatic mesh of triangular shape is used. The profiles of displacement components are shown in Fig. 15. The average errors (as defined in the previous problem) in computation of the displacement components are found to be almost absolute zero (less than $1 \times 10^{-10}$ ). This demonstrates that the present procedure is able to predict the linear solutions almost exactly. Although the results not reported here, for the second case when all surfaces of the cube are fixed, the errors in computation of normal stress components are found to be almost absolute zero.

\section{Free Plate with Temperature Variation through the Thickness Alone}

For a plate of arbitrary planform and of constant thickness which is completely free of surface traction and in which the temperature varies through the thickness alone, the analytical solutions for displacement and stress fields are available [26]. Any arbitrary planform of the plate can be chosen. A hexagonal planform of the plate is considered along with an initial mesh as shown in Fig. 16. One eighth of the domain is used for computations due to symmetry. A mesh consisting of non-uniform and skewed triangular prismatic cells is used. The heating of the plate is assumed by introducing a uniform volumetric heat source within the plate and assuming the top and bottom surfaces of the plate at constant temperature $T_{0}$ and rest of the surfaces of the plate perfectly insulated. This results into the temperature variation through the thickness alone. The analytical solutions are given by

$$
\begin{gathered}
T-T_{r}=T_{0}+\frac{q}{2 k_{s}}\left(B^{2}-z^{2}\right), \\
\delta u=\alpha_{s}\left(T_{0}+\frac{q}{k_{s}} \frac{B^{2}}{3}\right) x, \\
\delta v=\alpha_{s}\left(T_{0}+\frac{q}{k_{s}} \frac{B^{2}}{3}\right) y \\
\delta w=\alpha_{s}\left(T_{0}+\frac{3-v}{1-v} \frac{q}{k_{s}} \frac{B^{2}}{6}\right) z-\alpha_{s} \frac{1+v}{1-v} \frac{q}{k_{s}} \frac{z^{3}}{6} .
\end{gathered}
$$


All stress components are zero except

$$
\sigma_{x x}=\sigma_{y y}=\frac{1}{2} \frac{\alpha_{s} E}{1-v} \frac{q}{k_{s}}\left(z^{2}-\frac{B^{2}}{3}\right)
$$

where $q$ is the source of heat per unit volume, $k_{s}$ is the thermal conductivity of the plate material and $B$ is the half thickness of the plate. The thickness of the plate is assumed to be $2 B=0.12$ $\mathrm{m}$ and the plate material is assumed to have the properties $E=2 \times 10^{11} \mathrm{~Pa}, v=0.3$ and $\alpha_{s}=$

$1 \times 10^{-5} \mathrm{~K}^{-1}$. The $q / k_{s}$ ratio is assumed to be $5 \times 10^{5} \mathrm{Km}^{-1}$. The temperatures $T_{0}$ and $T_{r}$ are assumed to be equal to zero. The mesh refinement in the $z$-direction is found to be sufficient for predicting accurate solutions, as expected, since the temperature is assumed to be varying in only the $z$-direction. The results for the meshes consisting of 3 and 12 cells along the $z$ direction (along half thickness of the plate) are reported here. First the thermal problem is solved to obtain the temperature field in the plate. The temperature profile as a function of $z$ is shown in Fig. 17 with an overlapping plot for the analytical solution. Now the displacement and stress fields in the plate due to the predetermined temperature field are determined. The displacement components $\delta u$ and $\delta v$ are found to be linear functions of $x$ and $y$ coordinates respectively and in good agreement with the analytical solutions. The profile of displacement component $\delta w$ as a function of $z$ is plotted in Fig. 18 with overlapping plot for the analytical solution. Similarly the profile of stress component $\sigma_{x x}$ as a function of $z$ is plotted in Fig. 19 with overlapping plots for the analytical solution. The profile of the stress component $\sigma_{y y}$ is found to be identical with that of $\sigma_{x x}$. The maximum errors (with respect to the analytical solutions) in the results are reported in Table 3.

\section{Conclusions}

A cell-centered unstructured mesh finite volume procedure is presented for fluid flow, heat transfer and solid-body stress analysis. The feature of the line structure at the center of each cell face enables the discretization of the transport terms to be performed in a structured grid-like manner so that the solution accuracy is not affected by the mesh topology. The transformation relations between the global Cartesian coordinates and the local curvilinear coordinates for 
each cell face are used for the evaluation of the Cartesian gradients at the face-centers, which eliminates the requirement of computation of second order gradients at the cell-centers. The accuracy of the present procedure is demonstrated by solving a few fluid flow and heat transfer as well as solid-body stress analysis test problems. The procedure is able to predict accurate solutions even on meshes consisting of skewed elements. 


\section{References}

[1] M. A. Wheel, A Mixed Finite Volume Formulation for Determining the Small Strain Deformation of Incompressible Materials, International Journal for Numerical Methods in Engineering, vol. 44 (12), pp. 1843-1861, 1999.

[2] M. A. Wheel, A Geometrically Versatile Finite Volume Formulation for Plane Elastostatic Stress Analysis, Journal of Strain Analysis for Engineering Design, vol. 31 (2), pp. 111116, 1996.

[3] C. J. Greenshields, G. P. Venizelos, and A. Ivankovic, A Fluid-Structure Model for Fast Brittle Fracture in Plastic Pipes, Journal of Fluids and Structures, vol. 14 (2), pp. 221-234, 2000.

[4] M. Schafer, I. Teschauer, L. Kadinski, and M. Selder, A Numerical Approach for the Solution of Coupled Fluid-Solid and Thermal Stress Problems in Crystal Growth Processes, vol. 24 (3), pp. 409-419, 2002.

[5] I. Demirdzic, and S. Muzaferija, Finite-Volume Method for Stress-Analysis in Complex Domains, International Journal for Numerical Methods in Engineering, vol. 37 (21), pp. 3751-3766, 1994.

[6] I. Demirdzic, and S. Muzaferija, and M. Peric, Benchmark Solutions of Some Structural Analysis Problems Using Finite-Volume Method and Multigrid Acceleration, International Journal for Numerical Methods in Engineering, vol. 40 (10), pp. 1893-1908, 1997.

[7] H. Jasak, and H. G. Weller, Application of the Finite Volume Method and Unstructured Meshes to Linear Elasticity, International Journal for Numerical Methods in Engineering, vol. 48 (2), pp. 267-287, 2000.

[8] I. Demirdzic, and S. Muzaferija, Numerical-Method for Coupled Fluid-Flow, HeatTransfer and Stress-Analysis using Unstructured Moving Meshes with Cells of Arbitrary 
Topology, Computer Methods in Applied Mechanics and Engineering, vol. 125, pp. 235255, 1995.

[9] I. Demirdzic, S. Muzaferija, and M. Peric, Advances in Computational Heat Transfer, Fluid Flow, and Solid Body Deformation using Finite Volume Approaches, in W. J. Minkowycs, and E. M. Sparrow, Advances in Numerical Heat Transfer, vol. 1, pp. 5996, Taylor \& Francis, 1997.

[10] D. J. Mavriplis, Revisiting the least-squares procedure for gradient reconstruction on unstructured meshes, 16th AIAA Computational Fluid Dynamics Conference, Orlando, Florida 23-26 June 2003, pp. 1399-1411..

[11] S. R. Mathur, and J. Y. Murthy, A Pressure-Based Method for Unstructured Meshes, Numerical Heat Transfer, Part B: Fundamentals, vol. 31 (2), pp. 195-215, 1997.

[12] S. Das, S.R. Mathur, and J.Y. Murthy, An unstructured finite-volume method for structureelectrostatics interactions in MEMS, Numerical Heat Transfer, Part B: Fundamentals, vol. 60 (6), pp. 425-451, 2011.

[13] L. Davidson, A pressure correction method for unstructured meshes with arbitrary control volumes, International Journal for Numerical Methods in Fluids, vol. 22 (4), pp. 265-281, 1996.

[14] A. Dalal, V. Eswaran, and G. Biswas, A finite-volume method for Navier-Stokes equations on unstructured meshes, Numerical Heat Transfer, Part B: Fundamentals, vol. 54 (3), pp. 238-259, 2008.

[15] P. Traore, Y. M. Ahopo, and C. Louste, A robust and efficient finite volume scheme for the discretization of diffusive flux on extremely skewed meshes in complex geometries, J. Comput. Phys., vol. 228, pp. 5148-5159, 2009.

[16] S. C. Xue, and G.W. Barton, A finite volume formulation for transient convection and diffusion equations with unstructured distorted grids and its applications in fluid flow simulations with a collocated variable arrangement, Comput. Meth. Appl. Mech. Eng., vol. 253, pp. 146-159, 2012. 
[17] A. W. Date, Solution of Transport Equations on Unstructured Meshes with Cell-Centered Colocated Variables. Part I: Discretization, International Journal of Heat and Mass Transfer, vol. 48 (6), 1117-1127, 2005.

[18] C. Michler, S. J. Hulshoff, E. H. van Brummelen, and R. de Borst, A Monolithic Approach to Fluid-Structure Interaction, Computers and Fluids, vol. 33, pp. 839-848, 2004.

[19] A.W. Date, Fluid Dynamical View of Pressure Checkerboarding Problem and Smoothing Pressure Correction on Meshes with Colocated Variables, International Journal of Heat and Mass Transfer, vol. 46, pp. 4885 - 4898, 2003.

[20] S. V. Patankar, Numerical Heat Transfer and Fluid Flow, Taylor Francis, 1980.

[21] M. H. Sadd, Elasticity:Theory, Applications, And Numerics, 2nd ed., Academic Press, Burlington, MA, 2009.

[22] T. H. Kuehn, and R. J. Goldstein, Experimental and Theoretical-Study of NaturalConvection in Annulus Between Horizontal Concentric Cylinders, Journal of Fluid Mechanics, vol. 74, pp. 695-719, 1976.

[23] K. C. Karki, K. M. Kelkar, P. S. Sathyamurthy, and S. V. Patankar, Accurate Solutions for Laminar Flow and Heat Transfer in a Channel with a Backward-Facing Step, in American Society of Mechanical Engineers, Heat Transfer Division, (Publication) HTD, vol. 222, pp. 35-43, 1992.

[24] B. N. Jiang, T. L. Lin, and L. A. Povinelli, Large-Scale Computation of Incompressible Viscous-Flow by Least-Squares Finite-Element Method, Computer Methods in Applied Mechanics and Engineering, vol. 114, pp. 213-231, 1994.

[25] S. Timoshenko, and J. N. Goodier, Theory of Elasticity, 2nd ed., McGraw-Hill Book Company Inc., New York, 1951.

[26] B. A. Boley, and J. H. Weiner, Theory of Thermal Stresses, John Wiley and Sons Inc., New York, 1962. 
Table 1: Expressions of $a_{\phi}, \Gamma_{\phi}$ and $S_{\phi}$ in Eq. (17)

\begin{tabular}{ccccc}
\hline & $\phi$ & $a_{\phi}$ & $\Gamma_{\phi}$ & $S_{\phi}$ \\
\hline \multirow{3}{*}{ FLUID } & 1 & 1 & 0 & 0 \\
& $u_{i}$ & 1 & $\mu_{f}$ & $-\left(\partial p / \partial x_{j}\right) \delta_{i j}+\partial\left(\mu_{f} \partial u_{j} / \partial x_{i}\right) / \partial x_{j}+B_{i, f}$ \\
& $T_{f}$ & 1 & $k_{f} / c_{P, f}$ & $Q^{\prime \prime \prime} / c_{P, f}$ \\
\hline \multirow{2}{*}{ SOLID } & $\delta u_{i}$ & 0 & $\mu_{s}$ & $\partial\left[\lambda_{s} \delta_{i j} \partial\left(\delta u_{l}\right) / \partial x_{l}-\delta_{i j}\left(2 \mu_{s}+3 \lambda_{s}\right) / \partial x_{j}\left(T_{s}-T_{r}\right)\right] / \partial x_{j}+B_{i, s}$ \\
& & & & $Q^{\prime \prime \prime}$ \\
\hline
\end{tabular}


Table 2: The problem of natural convection in a horizontal concentric annulus: $\mathrm{k}_{\mathrm{eq}}$

\begin{tabular}{ccccc}
\hline $\mathrm{Ra}$ & \multicolumn{2}{c}{$\mathrm{k}_{e q}$ - inner wall ( mean value) } & \multicolumn{2}{c}{$\mathrm{k}_{e q}$ - outer wall ( mean value) } \\
& Present work & Kuehn and Goldstein [22] & Present work & Kuehn and Goldstein [22] \\
$10^{2}$ & 1.010 & 1.000 & 1.010 & 1.002 \\
$10^{3}$ & 1.096 & 1.081 & 1.098 & 1.084 \\
$10^{4}$ & 2.037 & 2.010 & 2.045 & 2.005 \\
\hline
\end{tabular}


Table 3: Maximum errors (percent) for the problem of free plate with temperature variation through the thickness alone

\begin{tabular}{lcc}
\hline & \multicolumn{2}{c}{ Maximum error, \% } \\
$\begin{array}{c}\text { 3 cells along } \\
\text { half thickness }\end{array}$ & $\begin{array}{c}\text { 12 cells along } \\
\text { half thickness }\end{array}$ \\
\hline$\delta w$ & 6.69 & 0.46 \\
$\sigma_{x x}$ & 22.75 & 0.95 \\
$\sigma_{y y}$ & 22.75 & 0.95 \\
\hline
\end{tabular}




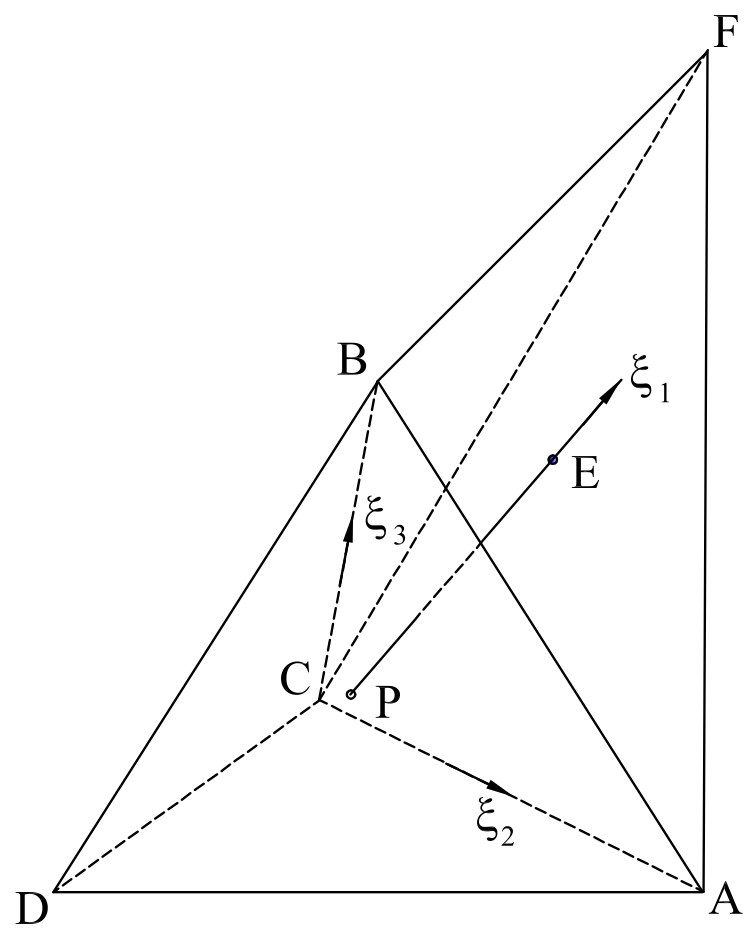

Figure 1: Local curvilinear coordinate system 


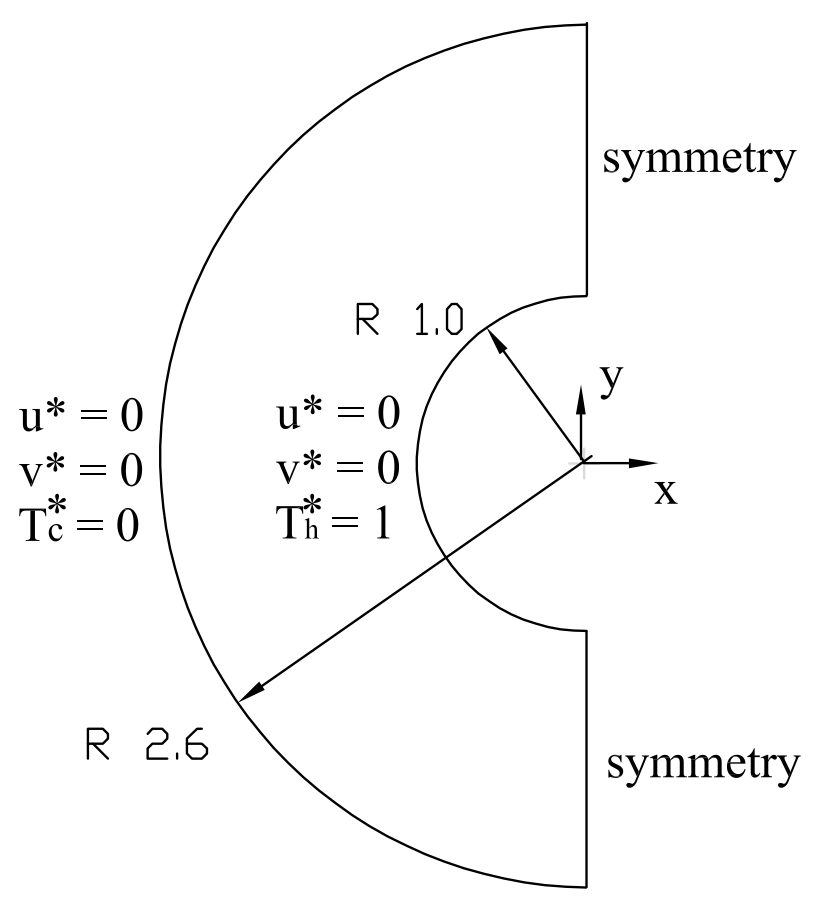

Figure 2: Schematic for the problem of natural convection in a horizontal concentric annulus 


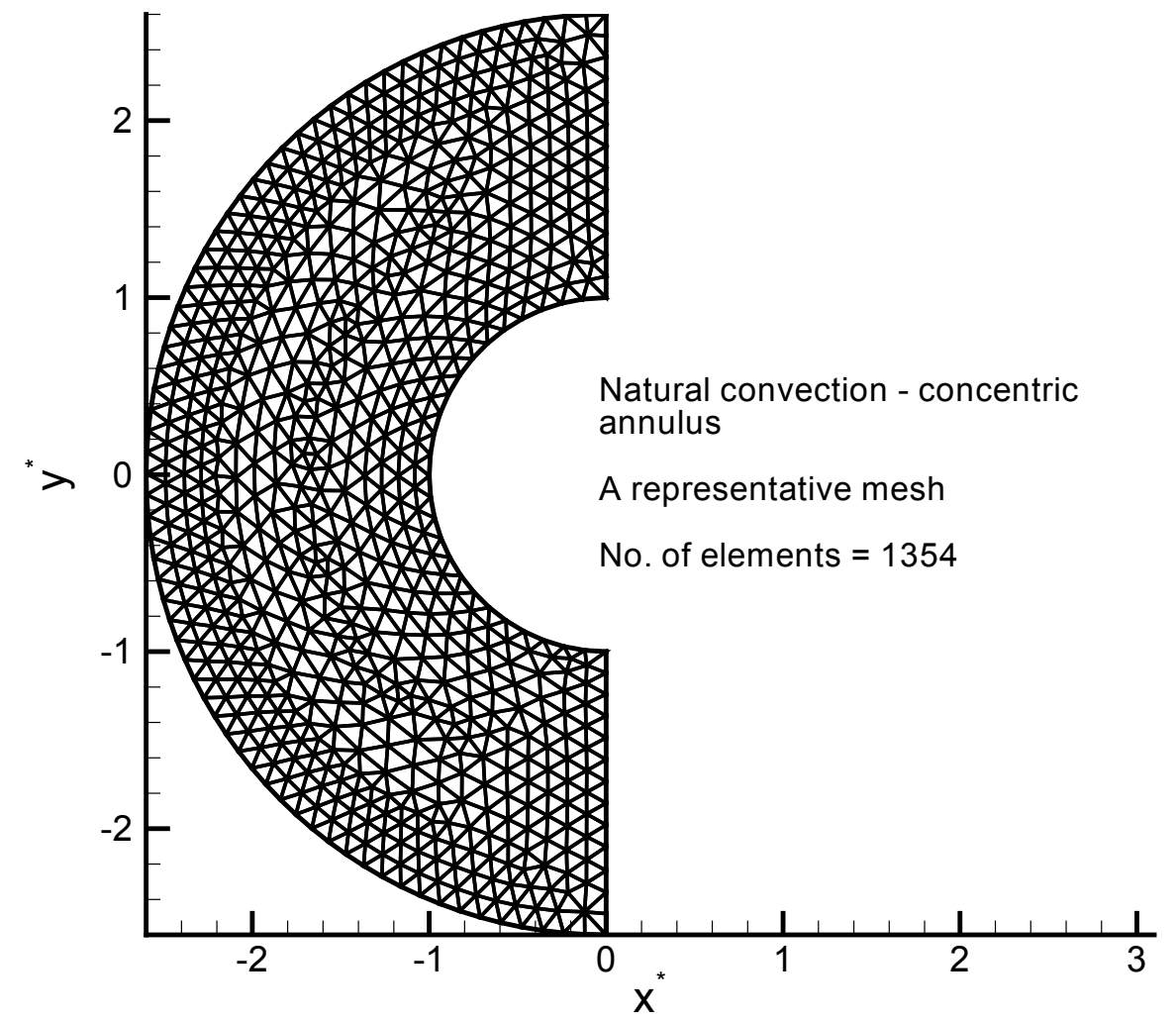

Figure 3: A representative mesh for the problem of natural convection in a horizontal concentric annulus 


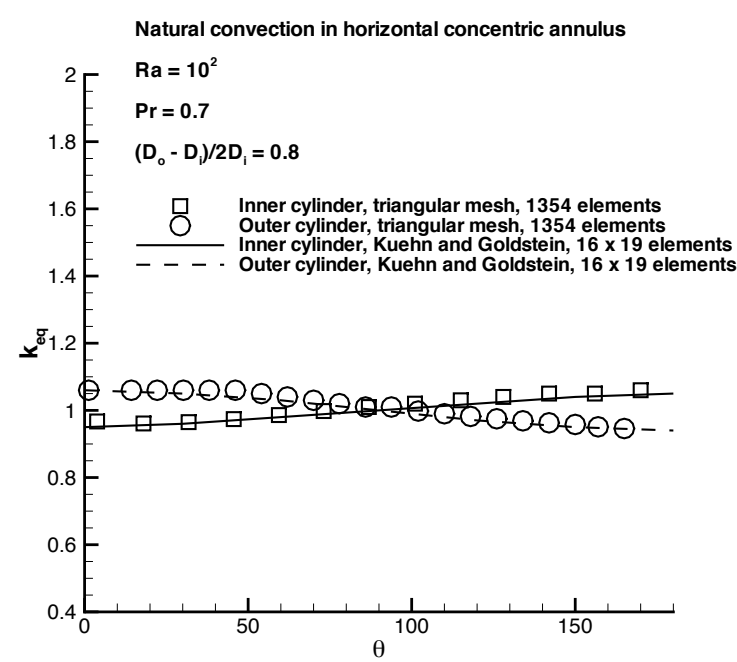

(a) $R a=100$

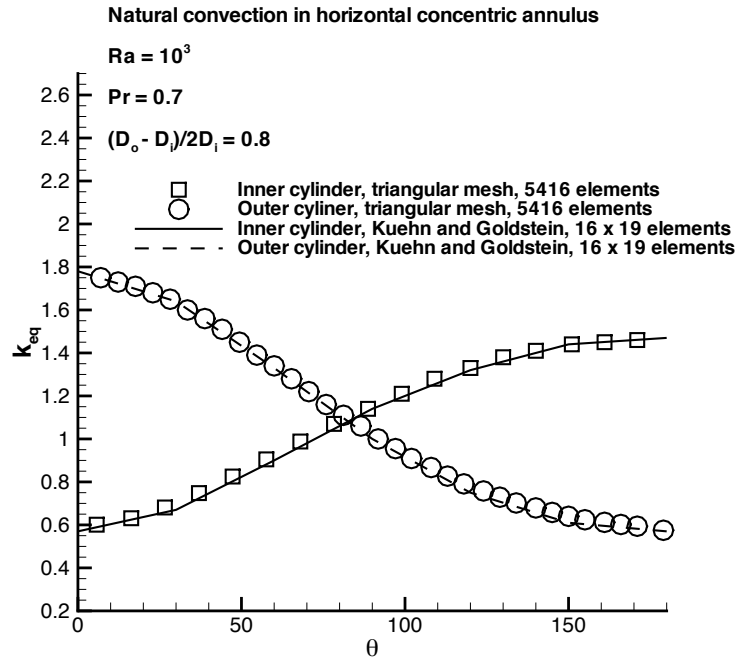

(b) $R a=1000$

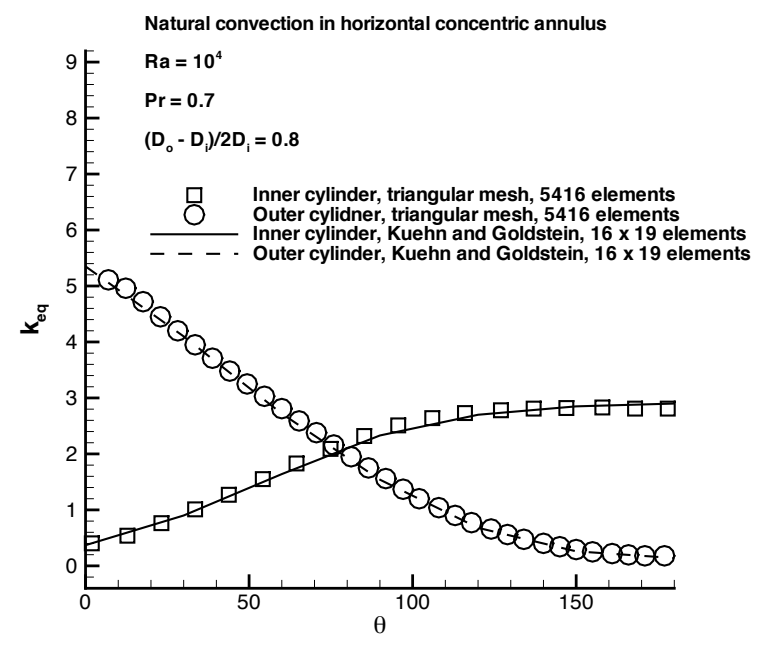

(c) $R a=10000$

Figure 4: Distribution of $k_{\mathrm{eq}}$ for the problem of natural convection in a horizontal concentric annulus 


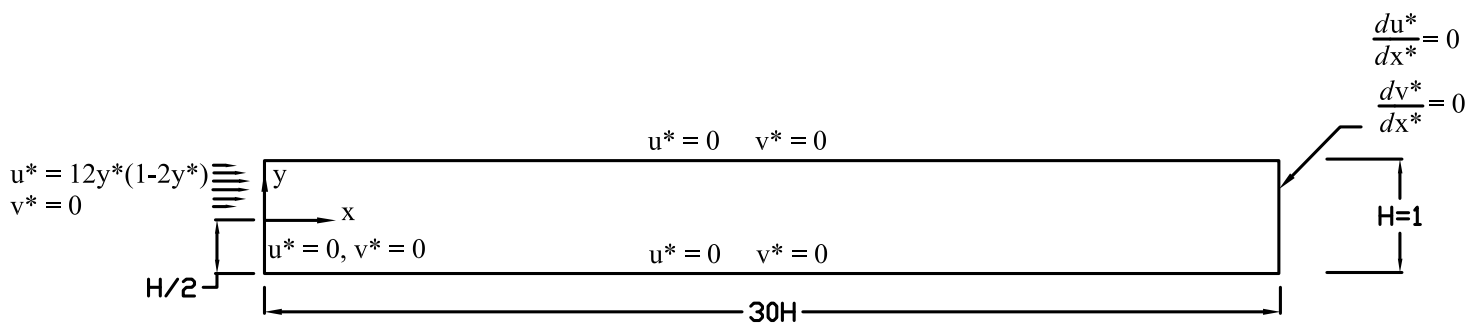

Figure 5: Schematic for the problem of flow over a backward facing step 


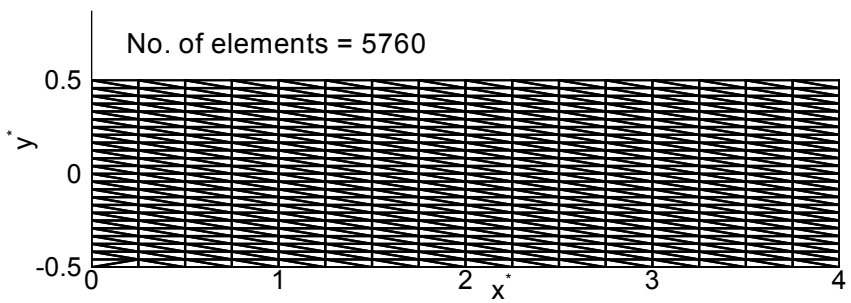

Figure 6: Mesh for the problem of flow over a backward facing step 


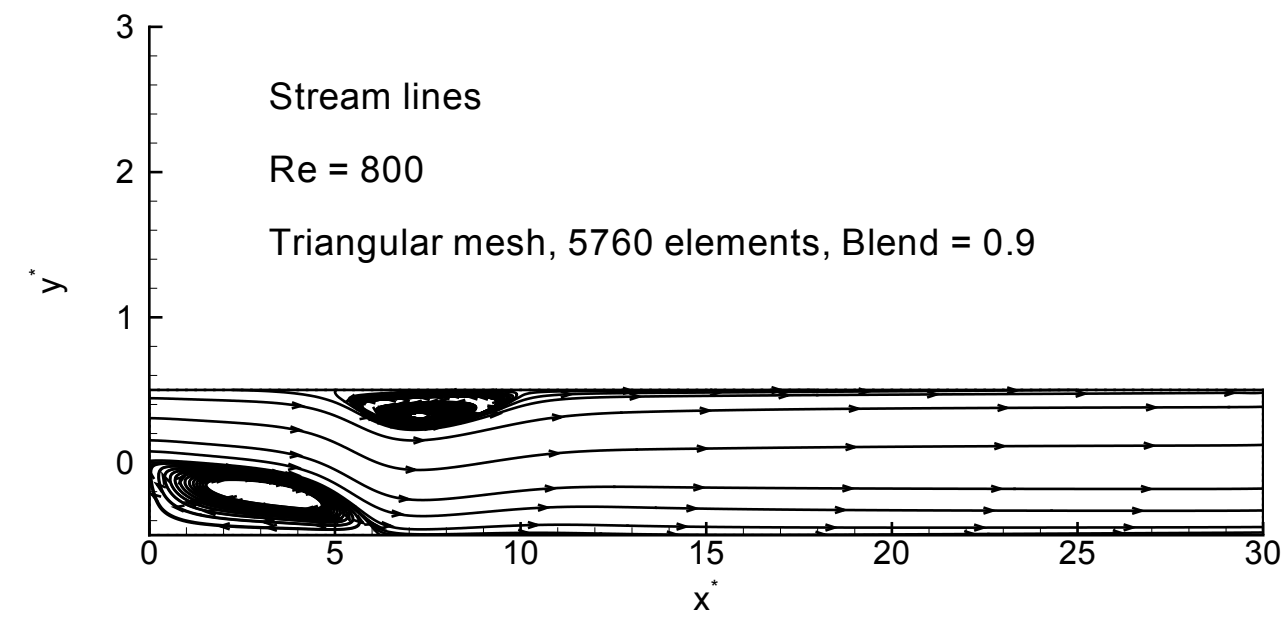

Figure 7: Streamlines for the problem of flow over a backward facing step 


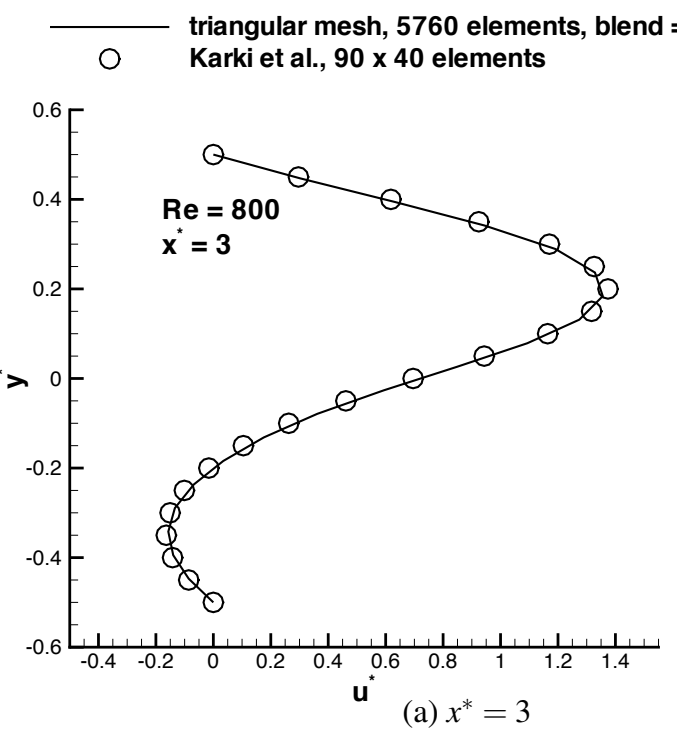

(a) $x^{*}=3$ triangular mesh, 5760 elements, blend $=0.9$ Karki et al., $90 \times 40$ elements

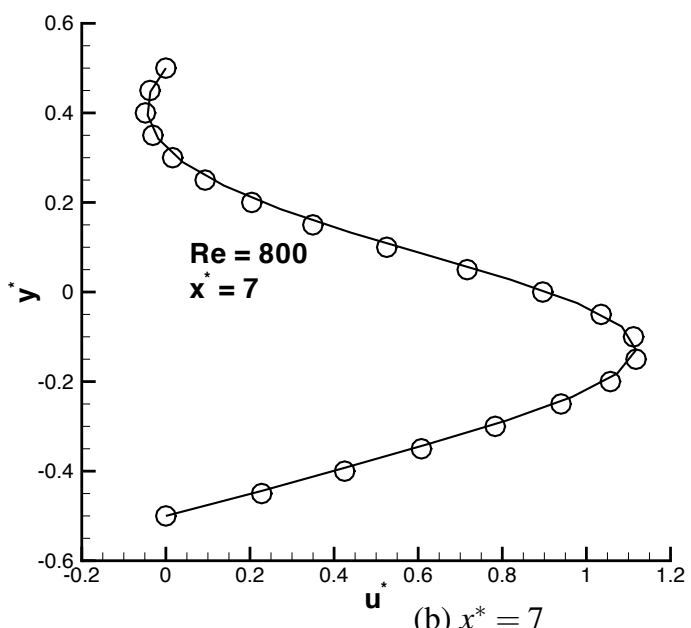

(b) $x^{*}=7$

triangular mesh, 5760 elements, blend $=0.9$ Karki et al., 90 x 40 elements
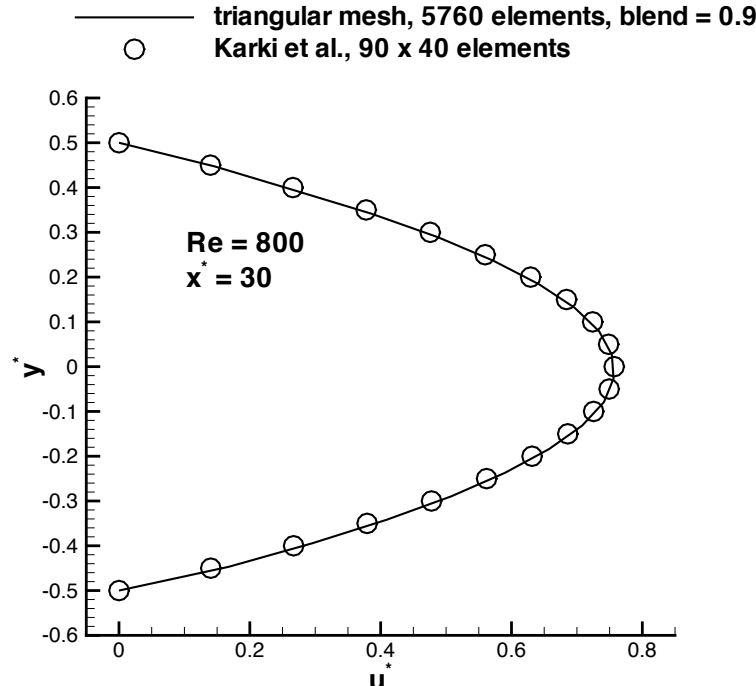

(d) $x^{*}=30$

Figure 8: Profiles for $x$ - velocity for the problem of flow over a backward facing step 


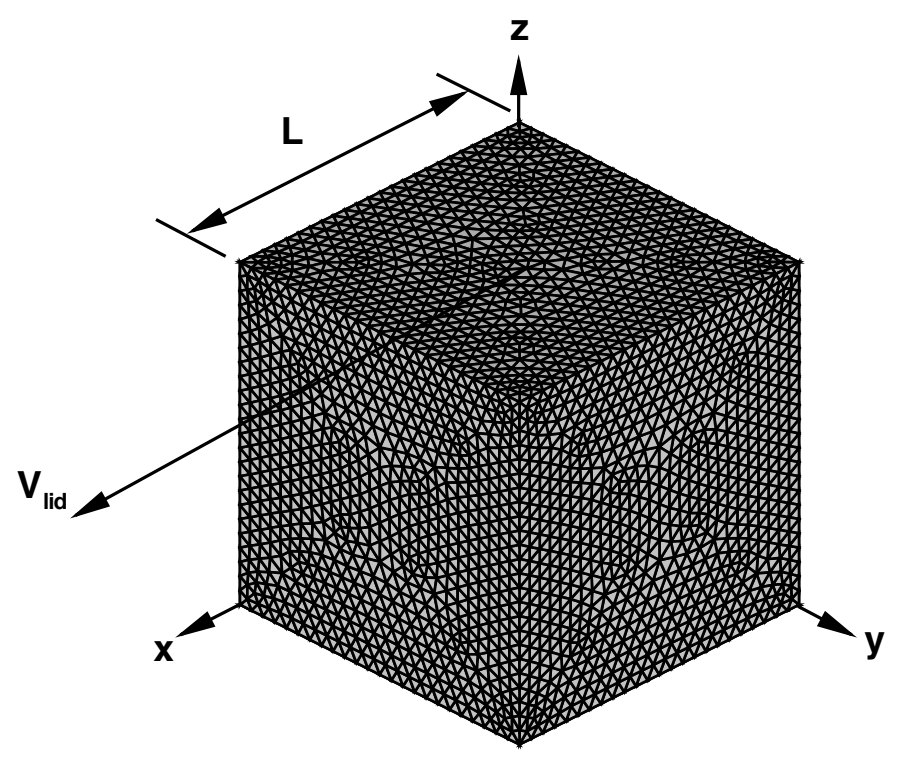

On the vertical walls and the bottom wall all velocity components are zero

Figure 9: Schematic and mesh for the problem of lid driven cubic cavity 


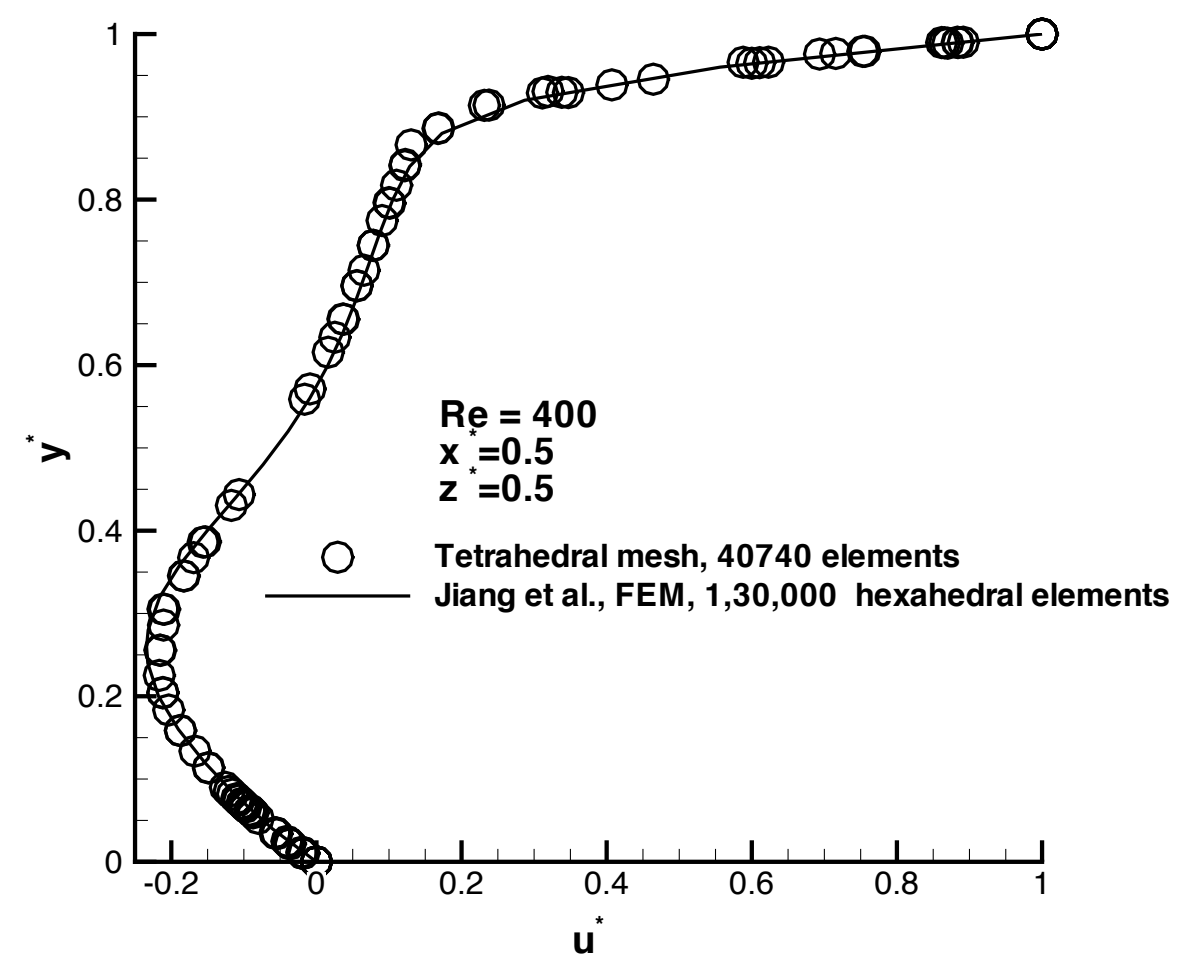

Figure 10: The problem of lid driven cubic cavity: $x$-velocity profile at $x^{*}=0.5$ and $z^{*}=0.5$ 


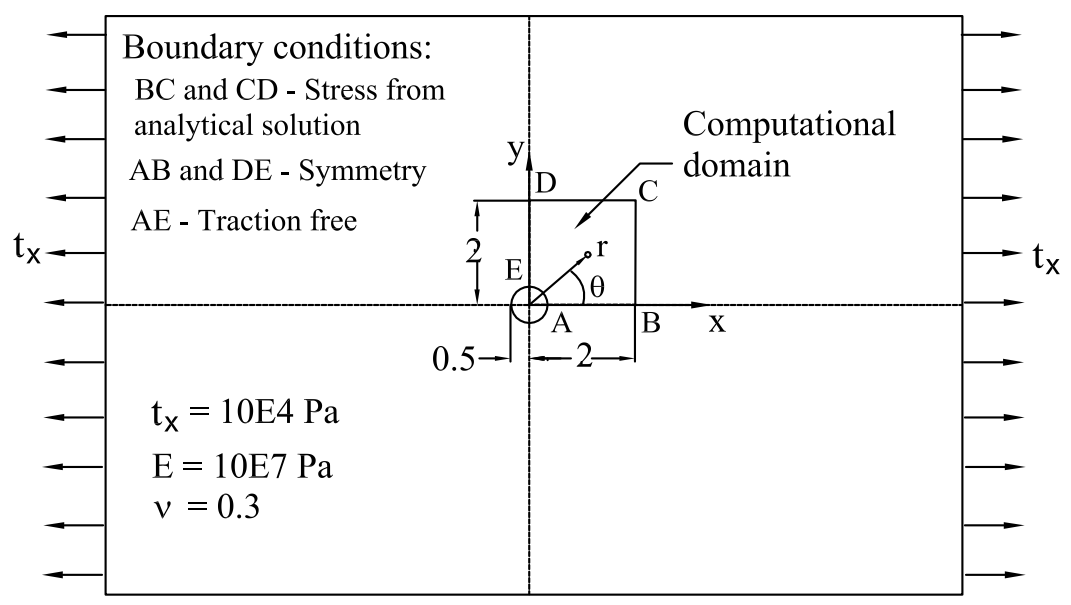

Figure 11: Schematic of the problem of flat plate with a circular hole subjected to uniform tension 


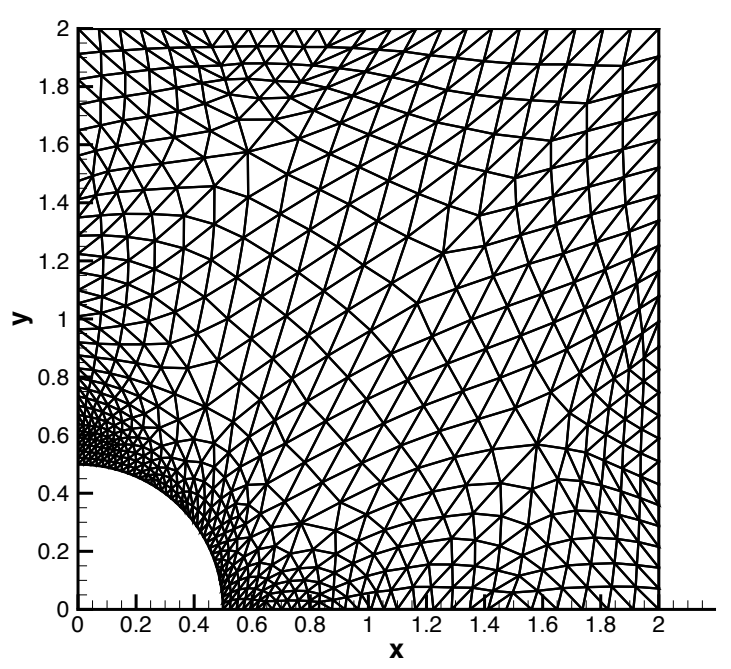

Figure 12: Representative mesh for the problem of flat plate with a circular hole subjected to uniform tension 


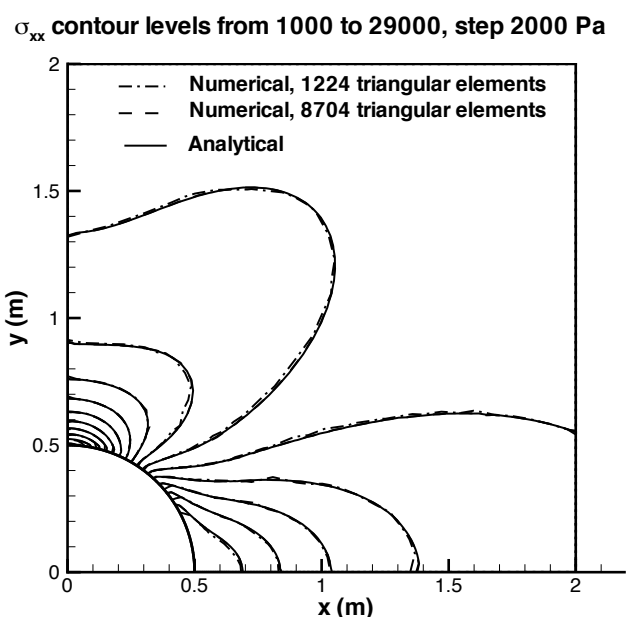

(a) $\sigma_{x x}$

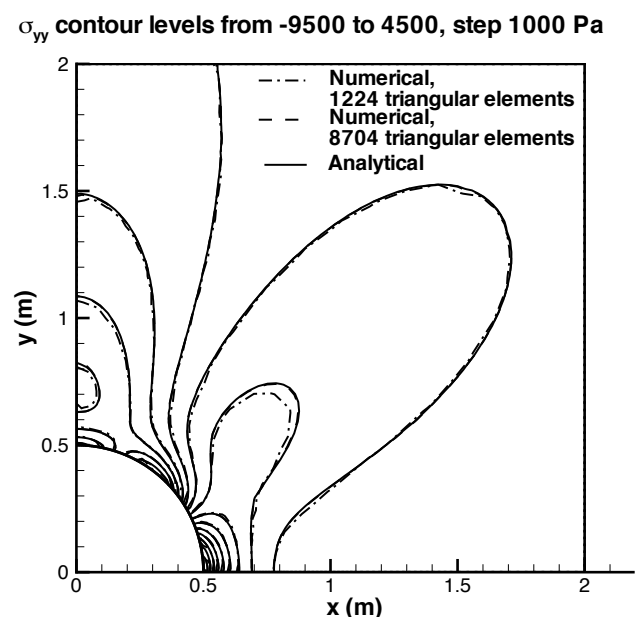

(b) $\sigma_{y y}$

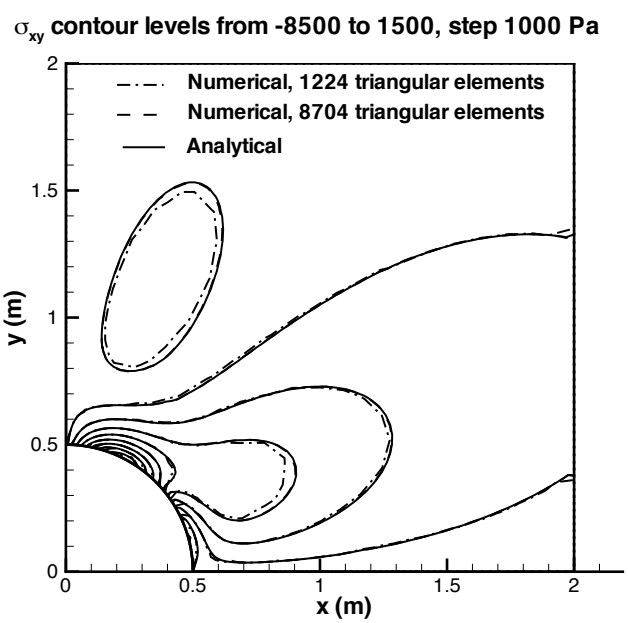

(c) $\sigma_{x y}$

Figure 13: Contour of stress components for the problem of of flat plate with a circular hole subjected to uniform tension 


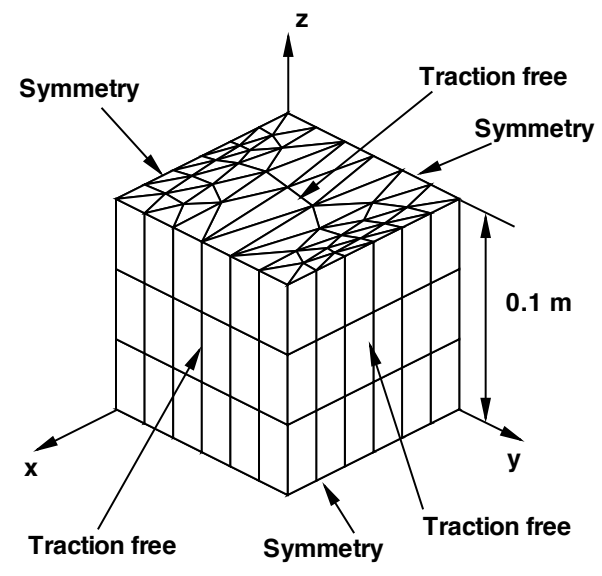

Figure 14: Schematic and mesh for the problem of a cube subjected to a uniform temperature rise 


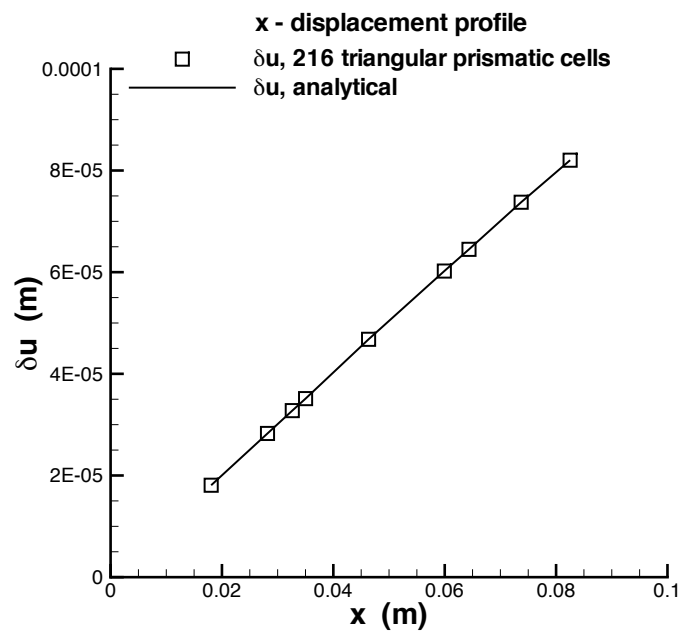

(a) $x$-displacements

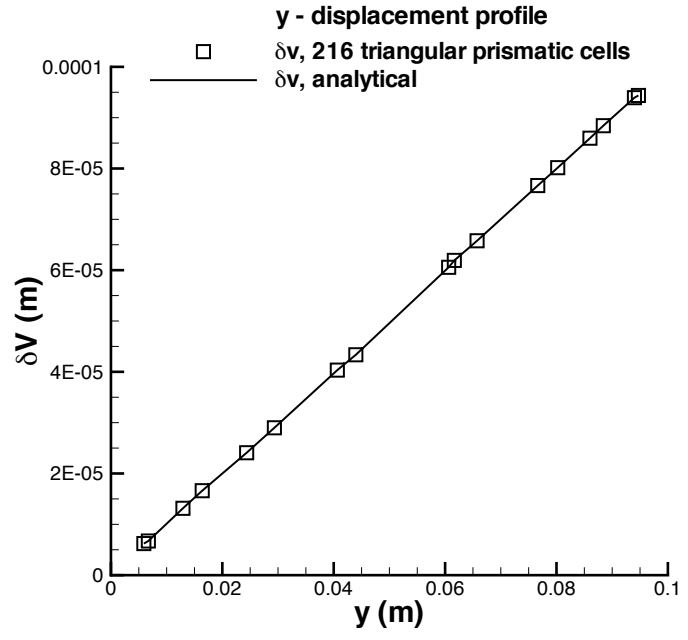

(b) $y$-displacements

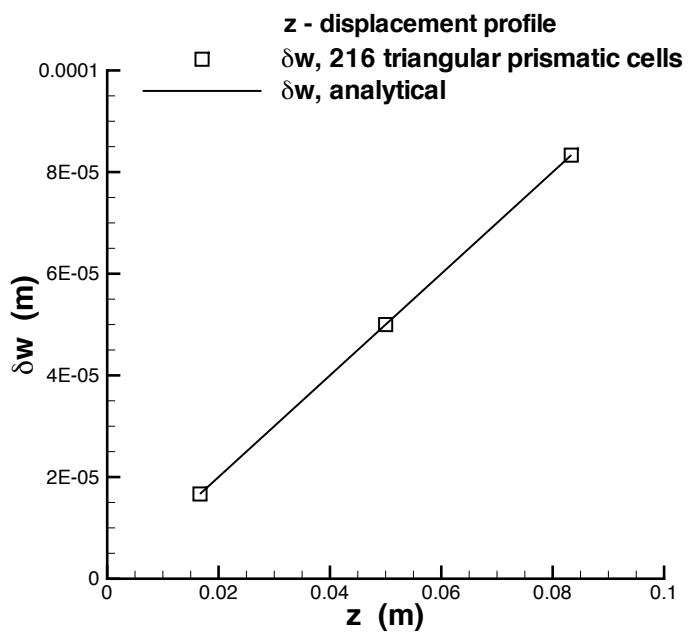

(c) $z$-displacements

Figure 15: Displacement profiles for the problem of a cube subjected to a uniform temperature rise, for traction free boundaries 


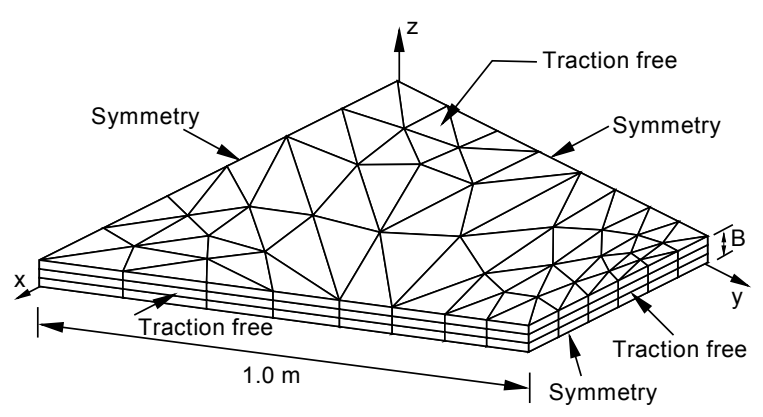

Figure 16: Schematic and mesh for the problem of free plate with a temperature variation through the thickness alone 


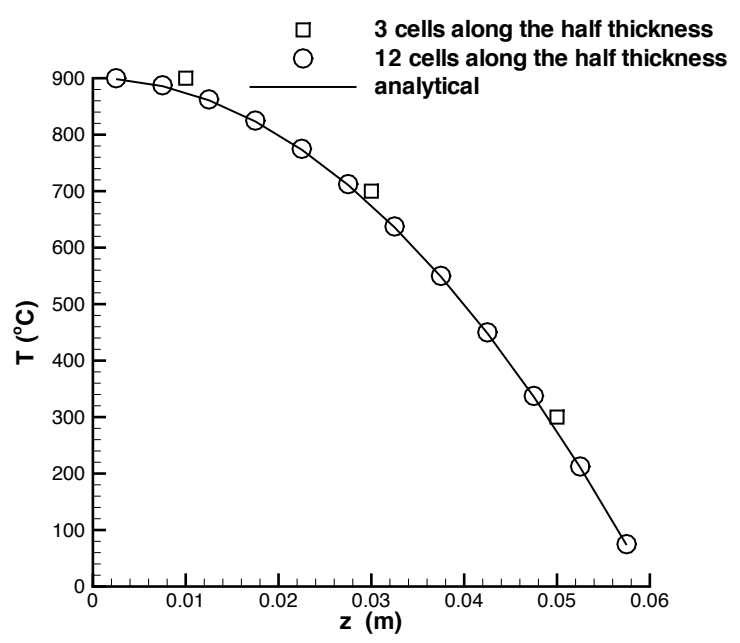

Figure 17: Temperature profile along $z$-axis for the problem of free plate with a temperature variation through the thickness alone 


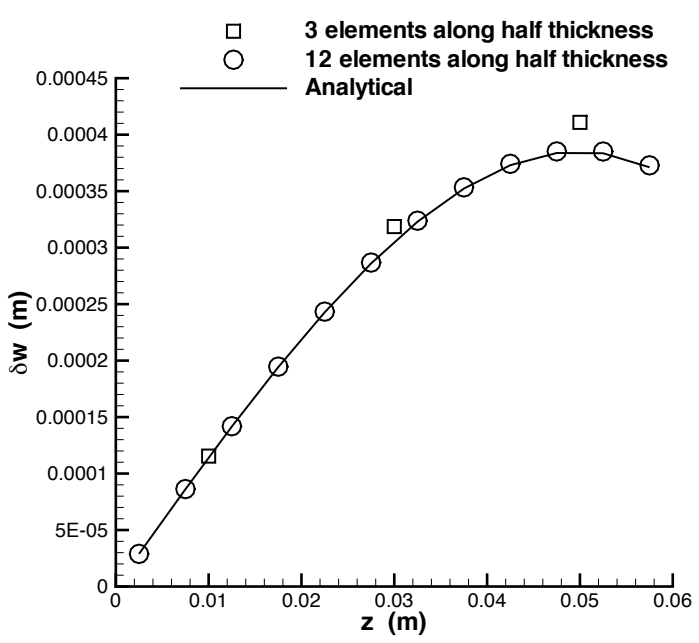

Figure 18: Profile of $z$-displacement for the problem of free plate with a temperature variation through the thickness alone 


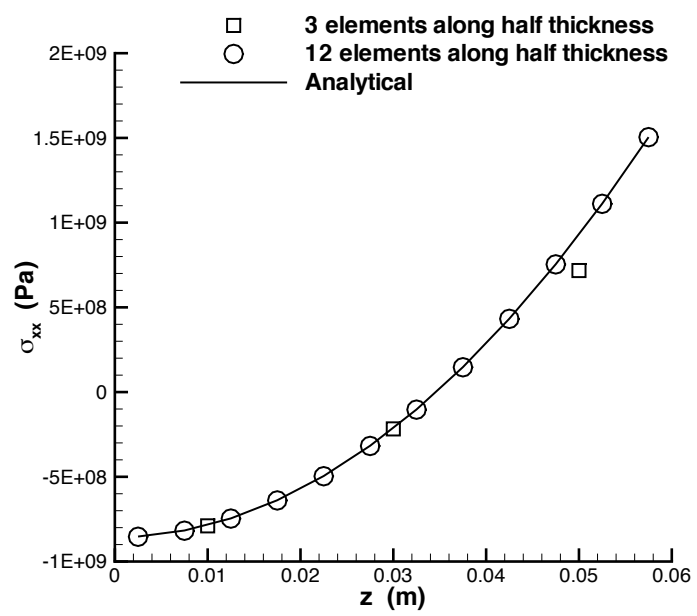

Figure 19: Profile of $\sigma_{x x}$ for the problem of free plate with a temperature variation through the thickness alone 